\title{
Tryptophan Metabolites in Irritable Bowel Syndrome: An Overnight Time-course Study
}

\author{
Robert L Burr, ${ }^{1,2}$ Haiwei Gu, ${ }^{3}$ Kevin Cain, ${ }^{2,4}$ Danijel Djukovic, ${ }^{3}$ Xinyu Zhang, ${ }^{3}$ Claire Han, ${ }^{1,5}$ Nini Callan, ${ }^{6}$ Daniel Raftery, ${ }^{3,5}$ and \\ Margaret Heitkemper ${ }^{1 *}$ \\ ${ }^{1}$ Department of Biobehavioral Nursing and Health Informatics, University of Washington, Seattle, WA, USA; ${ }^{2}$ Office for Nursing Research, \\ University of Washington, Seattle, WA, USA; ${ }^{3}$ Northwest Metabolomics Research Center, Department of Anesthesiology and Pain Medicine, \\ University of Washington, Seattle, WA, USA; ${ }^{4}$ Department of Biostatistics, University of Washington, Seattle, WA, USA; ${ }^{5}$ Public Health Sciences \\ Division, Fred Hutchinson Cancer Research Center, Seattle, WA, USA; and ${ }^{6}$ Helfgott Research Institute, National University of Natural Medicine, \\ Portland, OR, USA
}

\section{Background/Aims}

Patients with irritable bowel syndrome (IBS) often report poor sleep quality. Whether poor sleep is associated with tryptophan (Trp) metabolites is unknown. We compared serum Trp metabolites in women with IBS and healthy controls (HCs) using targeted liquid chromatography mass spectrometry (LC-MS)-based profiling. In IBS only, we explored whether Trp metabolites are associated with IBS symptoms and subjective and objective sleep indices, serum cortisol, plasma adrenocorticotropic hormone (ACTH), and cortisol/ACTH levels.

\section{Methods}

Blood samples were obtained every 80 minutes in 21 HCs and 38 IBS subjects following an anticipation-of-public-speaking stressor during a sleep laboratory protocol. Subjects completed symptom diaries for 28 days. Adjacent values of metabolites were averaged to represent 4 time-periods: awake, early sleep, mid-sleep, and mid-to-late sleep. Thirteen of 20 targeted Trp metabolites were identified.

\section{Results}

Ten of 13 Trp metabolites decreased across the night, while nicotinamide increased in both groups. A MANOVA omnibus test performed after principal component analysis showed a significant difference in these 13 principal component $(P=0.014)$ between groups. Compared to HCS, nicotinamide levels were higher and indole-3-lactic acid levels lower in the IBS group. Melatonin and indole-3-acetic acid levels were associated with several subjective/objective sleep measures; decreased stool consistency/frequency and abdominal pain were positively associated with melatonin and serotonin in the IBS group. The kynurenine and kynurenic acid were associated with ACTH (positively) and cortisol/ACTH (negatively).

\section{Conclusions}

Nighttime Trp metabolites may provide clues to poor sleep and stress with IBS. Further study of the mechanism of metabolite action is warranted.

(J Neurogastroenterol Motil 2019;25:551-562)

\section{Key Words}

Irritable bowel syndrome; Melatonin; Sleep; Tryptophan

Received: March 5, 2019 Revised: June 1, 2019 Accepted: July 20, 2019

(a) This is an Open Access article distributed under the terms of the Creative Commons Attribution Non-Commercial License (http://creativecommons. org/licenses/by-nc/4.0) which permits unrestricted non-commercial use, distribution, and reproduction in any medium, provided the original work is properly cited.

*Correspondence: Margaret Heitkemper, PhD Department of Biobehavioral Nursing and Health Informatics, School of Nursing, University of Washington, 1959 NE Pacific Street, Seattle, WA 98195, USA

Tel: +1-206-543-1091, Fax: +1-206-543-4771, E-mail: heit@uw.edu 


\section{Introduction}

Globally, approximately $10-20 \%$ of adults are diagnosed with irritable bowel syndrome (IBS). As such, IBS exerts a significant economic, social, and emotional burden. ${ }^{1}$ IBS is a heterogeneous condition characterized by abdominal pain and alterations in bowel functions and it occurs disproportionately in women. IBS is associated with a number of other symptoms such as poor sleep, fatigue, and muscle aches. Approximately $25-55 \%$ of persons with IBS report problematic sleep. ${ }^{2}$ However, discovery of a specific physiological link between sleep quality or duration and symptoms of IBS remains elusive.

We previously reported that women with IBS report more sleep disturbances including poor sleep quality, awakening during the night, difficulty getting to sleep, and "feeling unrefreshed" in the morning when compared to healthy control (HC) women. ${ }^{3}$ Using daily symptom diaries, we found that poor sleep the night before is correlated with increased abdominal pain severity the next day. In one study, women with IBS had higher levels of plasma cortisol during the early part of the night as compared to $\mathrm{HC}$ women. ${ }^{4} \mathrm{Ex}-$ posure to a social stressor, ie, anticipation of public speaking, prior to sleep exaggerated this difference. ${ }^{5}$

Cortisol influences a number of metabolic processes including the induction of tryptophan (Trp) 2,3-dioxygenase in the liver. ${ }^{6}$ The metabolism of $\operatorname{Trp}$ to the kynurenine pathway and production of subsequent metabolites such as kynurenic acid, hydroxykynurenine, quinolic acid, and nicotinamide is increased by stress and cortisol. ${ }^{7}$ Whether the stress-induced increase in the Trp-kynurenine pathway results in reduced production of serotonin or melatonin is unknown. ${ }^{8}$ It can be conjectured that an increase in Trp metabolism to the kynurenine pathway could potentially reduce the amount of Trp available for serotonin-melatonin production. ${ }^{9}$

In our prior study in women with IBS, we examined associations of tryptophan metabolites with subjective and objective sleep measures at one time point during the night (ie, 1 hour after the onset of sleep) using a non-targeted liquid chromatography mass spectrometry (LC-MS) approach. ${ }^{10}$ In this study, we found that the melatonin was associated with sleep onset, suggesting reductions in this Trp-serotonin-melatonin pathway could account for reports of delayed sleep onset in IBS. ${ }^{10}$ Pharmacological dosing with melatonin is also associated with decreased visceral sensitivity and fewer reports of abdominal pain in IBS. ${ }^{11,12}$ Serotonin and other Trp metabolites have roles in gastrointestinal (GI) motility and visceral sensitivity. Thus, stress and subsequent increases in cortisol levels could be associated with IBS symptoms and sleep alterations via Trp metabolite pathways.

Studies of Trp metabolites and their effects are beginning to emerge in other chronic diseases such as autism. ${ }^{13,14}$ In murine models, Trp metabolites are associated with behaviors reflective of depression, cognitive difficulties, and fatigue. ${ }^{15-17}$ Intestinal bacteria contribute to the production of Trp as well as the conversion of dietary $\operatorname{Trp}$ to indole-3-acetic acid and indole-3-lactic acid. These metabolites are converted to tryptamine and other metabolites in the liver. The potential associations of these metabolites to sleep and symptoms remain unexplored in IBS.

Therefore, using a targeted LC-MS analysis we extend our previous research with this sample, ${ }^{10}$ by exploring Trp metabolites at 4 time points during the night, with additional metabolites (ie, nicotinic acid, tryptamine, indole, indole pyruvate, indole-3-acetic acid, and indole-3-lactic acid). Furthermore, we examined relationships between Trp metabolites and sleep and IBS symptoms over the night. This will provide a comprehensive understanding about the patterns of Trp metabolites and its relationships with sleep and IBS symptoms.

The purpose of the current study is to describe and compare the patterns of Trp metabolites over the night in women with IBS and HCs exposed to a social stressor in a university sleep laboratory. For exploratory aims in the IBS group correlational analyses were performed. First, we explored the association of Trp metabolites with symptoms, stool characteristics, and subjective/objective sleep indices. Second, we tested whether cortisol, adrenocorticotropic hormone (ACTH), and the cortisol/ACTH ratio levels are related to Trp metabolites.

\section{Materials and Methods}

\section{Design}

This metabolomic study utilized blood samples collected during a study of stress and sleep in women with IBS and HCs. LCMS was used to detect nighttime Trp metabolites in serum samples collected during the third night of a 3-day sleep laboratory protocol (8:00 pm to 6:00 am).

\section{Subjects and Setting}

Community advertisements were used to recruit women with IBS and HCs (ages 18-45 years). All women were initially screened by telephone. The details regarding recruitment and screening have been published elsewhere. ${ }^{5}$ For those in the IBS group, women 
had to have a diagnosis for at least 6 months made by a health care provider and based on the Rome III criteria. The Rome III criteria requires that over the preceding 3 months subjects had to have abdominal discomfort or pain more than $25 \%$ of the days that was associated with 2 of 3 features: (1) relieved with defecation, (2) onset associated with a change in frequency of stool, or (3) onset associated with a change in form (appearance) of stool. The Rome III Diagnostic Questionnaire for Functional GI Disorders Rome III criteria was used to confirm the diagnosis. Women whose symptoms were aligned with IBS-mixed were excluded. For those women in the HC group, they could not have a history of functional GI disorders or serious health conditions. In both groups women were excluded if they were currently taking medications for GI symptoms; had a history of an organic GI disease, or urinary tract and/or gynecological pathology; were currently using daily medications, eg, tricyclic antidepressants, that could alter serotonin or cortisol; were obese, ie, a body mass index $\left(\mathrm{kg} / \mathrm{m}^{2}\right)>35$; were employed during the late evening or at night; reported a primary sleep disorder; had a history of severe co-morbid pain or mental health conditions; or consumed beverages containing $\geq 300 \mathrm{mg}$ of caffeine in the afternoon/evening, or drank 3 or more alcohol-containing beverages per day.

For the current study, 472 serum samples from IBS patients (n $=38)$ and HCs $(\mathrm{n}=21)$ collected at 8 time points over the night were analyzed.

\section{Procedures}

Prior to recruitment, the study was reviewed and approved by the University of Washington Human Subjects Committee (059418-VO1) annually, and we obtained informed consent from the participants. Women gave consent, completed baseline questionnaires including demographics, Rome questionnaire, daily diaries, recalled sleep quality, underwent physical assessment, reviewed the data collection procedures, and toured the Sleep Laboratory. Women filled in a daily diary over 1 menstrual cycle.

\section{Sleep laboratory protocol}

The sleep laboratory protocol has been described elsewhere. ${ }^{5,10}$ For all women, sleep testing was performed during the mid-luteal menstrual cycle phase, which was validated using a urine test for luteinizing hormone (ClearPlan Easy; Unipath Diagnostics Inc, Waltham, MA, USA). The sleep study was scheduled for the third week of their pill pack for women taking oral contraceptives. All participants were asked to avoid drinking caffeine-containing beverages, taking aspirin, acetaminophen, or non-steroidal anti- inflammatory drugs within 6 hours of their usual bedtime. Participants were asked to refrain from drinking alcohol-containing drinks during the 3-day sleep study. Monetary compensation was given at the end of the protocol.

To minimize environmental effects of sleep laboratory on our outcome variables, participants had an "adaptation" and a "baseline" night in the sleep laboratory to acclimate them to sleep laboratory and equipment. Thus, women slept in the research sleep laboratory for 3 consecutive nights ("adaptation," "baseline," and "stress"). During the "adaptation" night, women were screened for periodic leg movement disorder and apnea/hypopnea using polysomnography (PSG). If present, the participant did not continue in the study. On the "baseline" night (second night), only PSG data were collected. On the third night, blood samples were obtained; ${ }^{5}$ an intravenous catheter was inserted at 08:00 PM and samples drawn every 20 minutes, aliquoted and stored at $-80^{\circ} \mathrm{C}$. On night 3 women were reminded that upon awakening they would be giving a public 10-minute talk on their experiences in the sleep laboratory. This 'public speaking' was used as the "social stressor," and was videotaped in a lecture room attended by research staff. For this metabolite study, samples collected every 80 minutes were used.

\section{Measures}

\section{Rome III questionnaire}

The participants completed the Rome III questionnaire for IBS. Its development and validation have been previously described. ${ }^{18}$

\section{Daily diary measures}

Every day for 4 weeks, participants filled in a daily diary. The 26 symptoms were rated on a scale of 0 (not present) to 4 (very severe). Each symptom rating was summarized as the mean rating over 28 days. Symptoms used included abdominal pain/discomfort, abdominal pain after eating, bloating, intestinal gas, constipation, and diarrhea. The diary also included "difficulty falling asleep," "waking up during the night," "waking too early," "sleep quality," and "adequacy of sleep," "feeling rested," and "daytime sleepiness." Participants recorded the clock times when they went to bed and when they awoke. To mitigate variations in weekday/weekend effects, estimates of typical sleep pattern were summarized as median monthly bedtime and time of wakening.

The consistency of each stool was rated using the following scale of 0 (no stool), 1 (very hard), 2 (hard), 3 (formed), 4 (loose) or 5 (watery) in the daily diary. ${ }^{5}$ These ratings were summarized 
as the mean consistency score over 28 days. The average number of stools per day and the percent of days with hard and loose stools were calculated.

\section{Recalled sleep quality}

The 19-item Pittsburgh Sleep Quality Index (PSQI) was used to measure subjective sleep quality, sleep latency, sleep duration, habitual sleep efficiency, sleep disturbances, use of sleeping medication, and daytime dysfunction. ${ }^{19}$ When a global PSQI score of greater than 5 is used to distinguish good from poor sleepers it has a diagnostic sensitivity of $89.6 \%$ and specificity of $86.5 \%(\kappa=0.75$, $P<0.001)^{20}$

\section{Polysomnography}

Objective sleep was measured using a standard PSG that included electroencephalogram, electromyogram, and electrooculogram. ${ }^{5}$ An Embla Recording Systems with Somnologica software 3.3.2 (Embla Systems, Thornton, CO, USA) recorded sleep. With a combined computer and human scoring protocol, 30 second epochs were scored using standard scoring criteria. Summary measures include sleep efficiency index, sleep onset latency, and rapid eye movement (REM), and percent time spent awake and in slow wave sleep stages 3 and 4 and in REM. In our laboratory, interrater agreement of $>90 \%$ was established for sleep scoring. ${ }^{21,22}$

\section{Serum sample preparation}

The serum samples were thawed at $4^{\circ} \mathrm{C}$ and then vortexed for 10 seconds. Fifty microliters of each sample were mixed with $50 \mu \mathrm{L}$ of $\mathrm{H}_{2} \mathrm{O} / \mathrm{MeOH}(50 / 50)$, containing ${ }^{13} \mathrm{C}$ labeled $\operatorname{Trp}(163.2 \mu \mathrm{M})$. Two hundred microliter $\mathrm{MeOH}$ were added to precipitate proteins, and then the mixture was incubated at $-20^{\circ} \mathrm{C}$ for 20 minutes. After centrifuging at $14000 \mathrm{rpm}$ for 20 minutes, $150 \mu \mathrm{L}$ of supernatant were collected and dried. Then each dried sample was reconstituted in $200 \mu \mathrm{L}$ of $\mathrm{H}_{2} \mathrm{O} / \mathrm{ACN}(75 / 25)$, containing $0.2 \%$ formic acid.

\section{Reagents}

LC-MS grade, ammonium acetate, acetonitrile, methanol, and acetic acid were purchased from Fisher Scientific (Pittsburgh, PA, USA). De-ionized water was provided by a Synergy Ultrapure Water System from EMD Millipore (Billerica, MA, USA). Standard compounds with purities of standards ranging from 95-99\% were purchased from Sigma-Aldrich (Saint Louis, MO, USA) or Fisher Scientific.

\section{LC-MS conditions}

The LC-MS/MS assays were done on an Agilent 1260 LC (Agilent Technologies, Santa Clara, CA, USA) AB Sciex QTrap 5500 MS (AB Sciex, Toronto, Canada) system. Twenty microliters of each sample were injected for analysis using positive ionization mode. Chromatographic separation was performed using a Waters XSelect HSS T3 column $(2.5 \mu \mathrm{m}, 2.1 \times 150 \mathrm{~mm})$. The flow rate was $0.3 \mathrm{~mL} / \mathrm{min}$. The mobile phase was composed of Solvents A ( $100 \% \mathrm{H}_{2} \mathrm{O}$ with $0.2 \%$ formic acid) and B (100\% ACN with $0.2 \%$ formic acid). After the initial 0.2 minute isocratic elution of $100 \% \mathrm{~A}$, the percentage of Solvent A decreased to $50 \%$ at $\mathrm{t}=10$ minutes. The composition of Solvent A further decreased to $5 \%$ at $\mathrm{t}=12$ minutes, and then the percentage of $\mathrm{A}$ remained the same $5 \%$ for 6 minutes $(\mathrm{t}=18$ minutes $)$. Thirteen metabolites including Trp, serotonin, melatonin, kynurenine, kynurenic acid, anthranilic acid, tryptamine, nicotinic acid, nicotinamide, indole, indole-acetic acid, indole-pyruvate, and indole-3-lactic acid were identified. The metabolite identities were confirmed by spiking the pooled serum sample used for method development with mixtures of standard compounds. The extracted MRM peaks were integrated using MultiQuant 2.1 software (AB Sciex). Trp metabolites not identified by this procedure included 3-hydroxyanthranilic acid, quinolinic acid, 5-hydroxyl-indole-3-acetic acid, 3-indole-sulfate, 5-hydroxytryptophan, xanthurenic acid, and 3-hydroxykynurenine.

\section{Cortisol and adrenocorticotropic hormone}

Blood was collected in silicone coated tubes, allowed to coagulate, then centrifuged. ${ }^{5}$ Chemiluminescence using the automated Immulite Analyzer (Diagnostic Products Co, Los Angeles, CA, USA) was used to measure serum cortisol levels. The intra-assay variation was $2.7 \%$ and the inter assay variation is $5.4 \%$. A separate sample was collected in iced EDTA tubes for ACTH. Plasma ACTH was assayed by chemiluminescence using the automated Immulite Analyzer (Diagnostic Products Co). For ACTH, the intra-assay variation was $6.2 \%$ and the inter assay variation was $9.4 \%$.

\section{Statistical Methods}

For each subject there were 104 measurements (13 metabolites each measured at 8 times). Because of the skewness of the data, $\log$ transformations were applied to all metabolites. We collapsed the 8 times into 4 time periods, by taking the average of the first two, followed by the average of the next two, etc. This produced 4 repeated measures that were referenced to 4 time periods of the night. The first period (T1) was the average of measures at 20:00 
and 21:20 (awake), 22:40 and 24:00 (T2; transition from wake to sleep), 01:20 and 02:40 (T3; early sleep), and 04:00 and 05:20 (T4; mid to late sleep). This compression of the within-subjects data was done to reduce the dimensionality, increase statistical stability, and preserve the temporal dynamics, without sacrificing interpretability.

The primary goal was to understand which if any metabolites differed between IBS and HCs, either in overall level or the pattern over the night. However, given the large number of measurements, the issue of multiple comparisons was addressed. The approach taken was to perform a single global omnibus test involving the multiple values over the night of all metabolites. If the omnibus test was significant, then we proceeded to analyze each metabolite separately and also explore combinations of metabolites.

For the first aim, descriptive plots were developed to graphically show the pattern of the 13 Trp metabolites over the night. As a second step of dimensionality reduction, PC analysis was applied to the 52 measures per person (13 metabolites multiplied by 4 time periods), and all $\mathrm{PC}$ with eigenvalue greater than one were retained for use in subsequent multivariate analyses.

We next performed an omnibus multivariate analysis by group (IBS vs HC). A MANOVA was used to test the null hypothesis that the means of all the retained PCs are the same in the IBS and HCs versus the alternative hypothesis that the means differ for one or more of these measures. A permutation test with 10000 replications was performed to validate the $P$-value from the MANOVA.

Two types of analyses were done for each of the 13 metabolites separately, a repeated measures ANOVA test of Group, Time, and Group-by-Time interaction, and a univariate test of the level of the metabolite at each of the 4 times separately.

Spearman rank correlation was used to examine associations of Trp metabolites with cortisol, ACTH, cortisol/ACTH, symptoms, stool characteristics and descriptors of subjective and objective sleep in the IBS group. For these analyses, metabolite values from the 4 times are summarized as 2 numbers: The mean of the 4 values, and the slope of a regression line fitted to the 4 values within each person which measures how much the metabolite level increases or

Table 1. Summary of Demographic and Clinical Characteristics of Women With and Without Irritable Bowel Syndrome

\begin{tabular}{|c|c|c|}
\hline & $\operatorname{HCs}(\mathrm{n}=21)$ & IBS $(\mathrm{n}=38)$ \\
\hline Age (mean $[\mathrm{SD}])[\min , \max ]$ & $27.9(5.6)[18,40]$ & $28.5(7.0)[18,44]$ \\
\hline \multicolumn{3}{|l|}{ Race/ethnicity ${ }^{\mathrm{a}}$ (n [\%]) } \\
\hline Caucasian & $17(81.0)$ & $35(92.1)$ \\
\hline Asian & $3(14.3)$ & $2(5.2)$ \\
\hline Black-African American & $1(4.8)$ & $0(0.0)$ \\
\hline Other & $0(0.0)$ & $1(2.6)$ \\
\hline Body mass index (mean [SD]) & $23.7(3.8)$ & $23.6(3.6)$ \\
\hline Married/partnered (n [\%]) & $5(23.8)$ & $7(18.4)$ \\
\hline College education (n [\%]) & $17(81.0)$ & $26(68.4)$ \\
\hline Oral contraceptive use (n [\%]) & $8(38.1)$ & $16(42.1)$ \\
\hline Current smoker (n [\%]) & $2(9.5)$ & $6(15.8)$ \\
\hline Drinks alcohol at least occasionally (n [\%]) & $18(85.7)$ & $32(84.2)$ \\
\hline \multicolumn{3}{|l|}{ Caffeine (n [\%]) } \\
\hline Coffee & $14(66.7)$ & $26(68.4)$ \\
\hline Tea & $14(66.7)$ & $24(63.2)$ \\
\hline Soda & $8(38.1)$ & $13(34.2)$ \\
\hline Abdominal pain ${ }^{\mathrm{b}}(\operatorname{mean}[\mathrm{SD}])$ & $1.92(5.54)$ & $22.32(17.09), P^{c}<0.001$ \\
\hline \multicolumn{3}{|l|}{ Baseline sleep measures (mean [SD]) } \\
\hline Pittsburgh Sleep Quality Index Global score & $3.60(1.85)$ & $4.37(1.97)$ \\
\hline Sleep efficiency index via polysomnography & $0.82(0.08)$ & $0.76(0.13), P^{c}=0.005$ \\
\hline Fragmentation index via polysomnography & $7.29(2.54)$ & $7.60(2.57)$ \\
\hline Diary: perceived adequacy of sleep & $2.84(0.45)$ & $2.42(0.57), P^{c}=0.003$ \\
\hline Diary: usual bedtime & $24: 15(1: 09)$ & $23: 47(1: 05), P^{c}=0.070$ \\
\hline Diary: usual wake up time & $08: 00(1: 24)$ & $07: 19(0: 55), P^{c}=0.045$ \\
\hline
\end{tabular}

${ }^{a}$ Hispanic $(13.2 \%, \mathrm{n}=5$ in irritable bowel syndrome [IBS] group, none in healthy controls [HCs]).

${ }^{\mathrm{b}} \%$ diary days with moderate to very severe abdominal pain.

${ }^{c} P$-value based on the independent $t$ test. Only significant $P$-values between the 2 groups were present. 
decreases over the night. Because of the numerous multiple comparisons/tests, results are considered exploratory/hypothesis generating and should be interpreted cautiously.

\section{Results}

\section{Demographics and Clinical Characteristics}

Demographic characteristics for the sample population are shown in Table 1. Age, race/ethnicity, or body mass index did not differ between HC and IBS groups. Both groups were predominantly white (81.0\% HCs:92.1\% IBS) and not married/partnered (76.2\% HCs:81.6\% IBS). Constipation-dominant IBS subtype was $52.6 \%(\mathrm{n}=20$ ), and Diarrhea-dominant IBS subtype was $47.4 \%$ ( =18) (data not shown). As expected the IBS group reported greater days with abdominal pain rated as moderate to severe. Although there were no group differences in PSQI scores, the IBS group had a reduced sleep efficiency index, worse daily perceived sleep, went to bed earlier and woke up earlier as compared to HCs (Table 1).

A
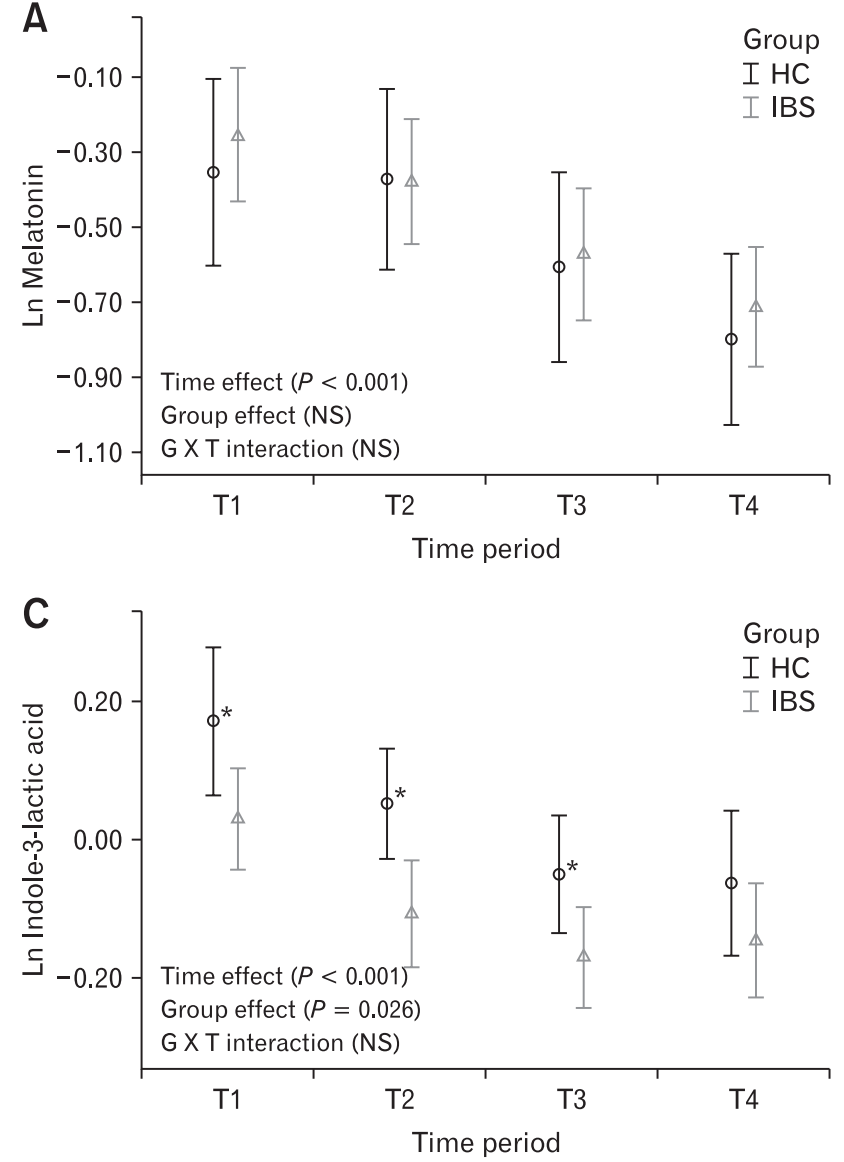

\section{Metabolites}

Description of time course across the night $(n=59)$

Ten of the 13 metabolites show a highly significant decrease over the night as seen in the example of melatonin in Figure 1A. The exceptions are serotonin and anthranilic acid which do not markedly change over the night, and nicotinamide which shows a significant increase over the night (Fig. 1B). The nighttime patterns of all 13 Trp metabolites are displayed in Supplementary Figure 1, and $P$-values for testing trend over time are shown in the first data column of Table 2.

\section{Irritable bowel syndrome and healthy control group differences in metabolites}

PC analysis on the 52 metabolite values (13 Trp metabolites multiplied by 4 time points) results in 13 PCs with eigenvalues greater than 1 . The initial omnibus test of IBS versus $\mathrm{HC}$ group differences using MANOVA on these $13 \mathrm{PC}$ s was significant at $P$ $=0.014$. The omnibus test result provides evidence that the IBS

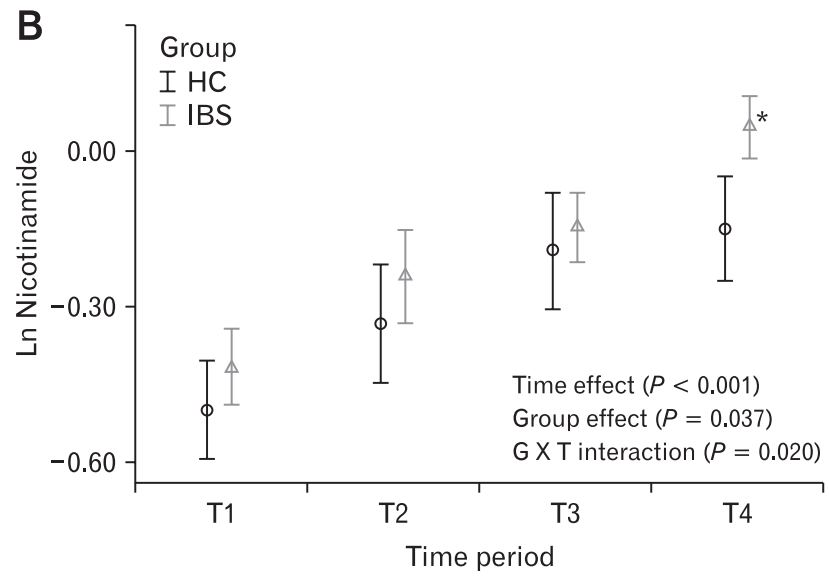

Figure 1. Exemplars of nighttime metabolites across 4 time periods between healthy controls ( $\mathrm{HCs}$ ) and women with irritable bowel syndrome (IBS). T1 represents 20:00 and 21:20 (awake); T2 represents 22:40 and 24:00 (transition from wake to sleep); T3 represents 01:20 and 02:40 (early sleep); and T4 04:00 and 05:20 (mid to late sleep). G, group; T, time; NS, not significant $(P>0.25)$. ${ }^{*}$ Group effect at each time point $(P<0.05)$. Y axis: log transformed metabolites. 
Table 2. Effects of Time and Group on Serum Metabolites in Women With and Without Irritable Bowel Syndrome

\begin{tabular}{|c|c|c|c|c|c|c|c|}
\hline \multirow{2}{*}{ Serum metabolites } & \multirow{2}{*}{ Time $^{a}$} & \multirow{2}{*}{$\begin{array}{l}\text { Main effect of } \\
\text { group }^{a}\end{array}$} & \multirow{2}{*}{$\begin{array}{l}\text { Group by time } \\
\text { interaction }\end{array}$} & \multicolumn{4}{|c|}{ Group effect at time $^{\mathrm{b}}$} \\
\hline & & & & Time 1 & Time 2 & Time 3 & Time 4 \\
\hline Metabolites & $\mathrm{df}=3$ & $\mathrm{df}=1$ & $\mathrm{df}=3$ & $\mathrm{df}=1$ & $\mathrm{df}=1$ & $\mathrm{df}=1$ & $\mathrm{df}=1$ \\
\hline Tryptophan & $<0.001$ & NS & NS & NS & NS & NS & NS \\
\hline Serotonin & 0.113 & 0.237 & NS & 0.156 & 0.199 & NS & NS \\
\hline Melatonin & $<0.001$ & NS & NS & NS & NS & NS & NS \\
\hline Kynurenine & $<0.001$ & 0.235 & NS & 0.124 & 0.128 & 0.247 & NS \\
\hline Kynurenic acid & $<0.001$ & 0.111 & NS & 0.231 & 0.110 & 0.039 & NS \\
\hline Anthranilic acid & 0.045 & NS & 0.196 & NS & 0.204 & NS & NS \\
\hline Nicotinic acid & $<0.001$ & NS & NS & NS & NS & NS & NS \\
\hline Nicotinamide & $<0.001$ & 0.037 & 0.020 & 0.163 & 0.211 & NS & 0.007 \\
\hline Tryptamine & $<0.001$ & NS & 0.173 & NS & NS & NS & NS \\
\hline Indole & $<0.001$ & NS & NS & NS & NS & NS & NS \\
\hline Indole pyruvate & $<0.001$ & 0.155 & NS & NS & 0.122 & NS & 0.198 \\
\hline Indole-3-acetic acid & $<0.001$ & 0.179 & NS & 0.123 & 0.229 & NS & 0.184 \\
\hline Indole-3-lactic acid & $<0.001$ & 0.026 & NS & 0.027 & 0.012 & 0.036 & 0.208 \\
\hline
\end{tabular}

${ }^{a} P$-values from repeated measures ANOVA that includes main effects of time, group, and group by time interaction.

${ }^{\mathrm{b}} P$-values from $t$ tests comparing means at each time point separately.

df, degrees of freedom of test effect; Time 1, awake; Time 2, early sleep; Time 3, mid-sleep; Time 4, early morning; NS, not significant ( $P>0.25$ ).

and HCs differ in their mean and/or pattern over the night for at least some metabolites and hence, it is appropriate to proceed with an examination of which metabolites differ and how, as seen in Table 2 and Figure 1 (all plots are shown in Supplementary Fig. 1).

As seen in Figure 1B, nicotinamide levels are higher in IBS and significantly different between the 2 groups at Time 4 (Table $2, P<0.01$ ). The main effect for group (difference in means) and group by time interaction (difference in pattern over the night) are both significant at $P=0.037$ and 0.020 , respectively.

Indole-3-lactic acid is lower in IBS than in HC (Fig. 1C). This difference is significant at Time 1 to Time 3 and the main effect for group is significant ( $P=0.026$; Table 2$)$. In a post-hoc analysis we combined indole-3-lactic acid and nicotinamide together to discriminate IBS from $\mathrm{HC}(P=0.004)$ as shown in Figure 2.

\section{Exploratory analyses of irritable bowel syndrome pain and bowel-related symptoms in irritable bowel syn- drome group}

Correlations of symptoms and sleep measures in the IBS group with mean and slope over the night of each metabolite are shown in Supplementary Tables 1 to 3 . Selected results are summarized here and in Supplementary Figure 2.

The slopes over the night of melatonin, nicotinic acid, and indole-3-acetic acid show a similar pattern of being positively correlated with abdominal pain, abdominal pain after eating, and

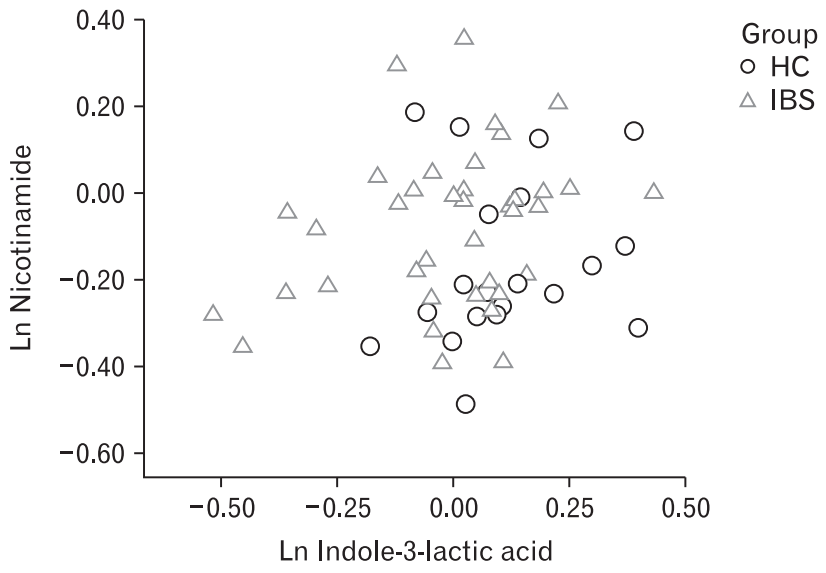

Figure 2. Data from 4 time period for nicotinamide and indole3-lactate are aggregated into whole night within-subject means, these 2 variables jointly separate irritable bowel syndrome (IBS) versus healthy controls (HCs) (logistic regression, $P=0.001$ ).

intestinal gas (Supplementary Table 1). Since these metabolites decrease during the night, positive correlation of metabolite slope with symptoms means that those with worse symptoms have a flatter pattern compared to those with less severe symptoms who have a steeper decrease. Trp, serotonin, and indole-3-lactic acid show similar positive associations with abdominal pain after eating. Figure 3A illustrates this pattern for Trp versus abdominal pain after eating.

The time-dependent level changes (slope) of serotonin posi- 

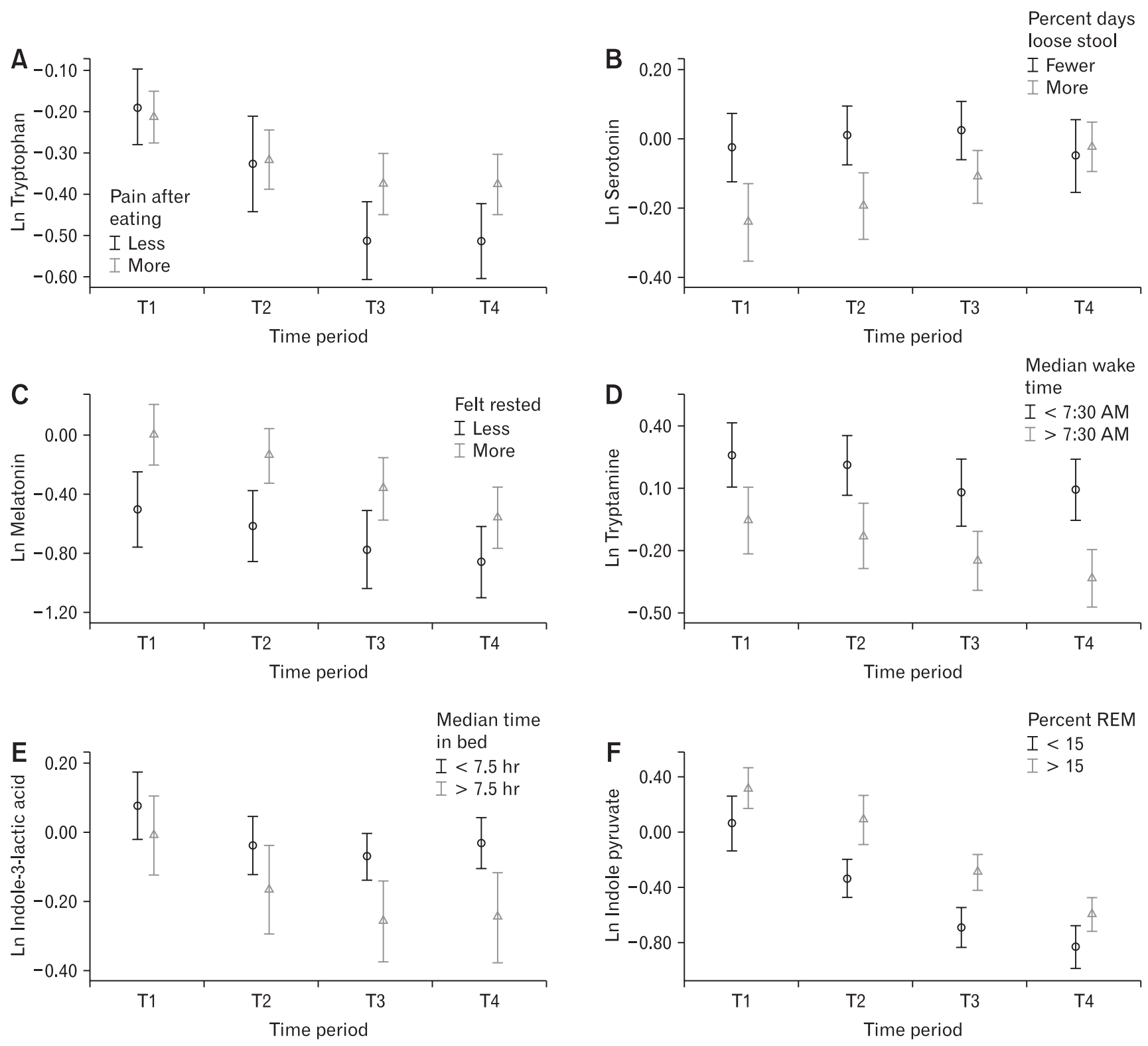

Figure 3. Exemplars of the relationship of selected tryptophan metabolites with daily diary symptoms in irritable bowel syndrome (IBS) patients. (A) Tryptophan based on median split of daily abdominal pain after eating, (B) serotonin based on median split of percent of days with loose stools, (C) melatonin based on median split of reported sleep quality across the month, (D) tryptamine based on median split of wake time reported in the daily diary, (E) indole-3-lactic acid based on median split of time in bed reported in the daily diary, and (F) indole-pyruvate based on median split of percent time in rapid eye movement (REM) sleep recorded by polysomnography. T1, 20:00 and 21:20 (awake); T2, 22:40 and 24:00 (transition from wake to sleep); T3, 01:20 and 02:40 (early sleep); T4, 04:00 and 05:20 (mid to late sleep).

tively correlated with worse diarrhea and looser and more frequent stools (Supplementary Table 1). This is illustrated in Figure 3B, where among those with more days with loose stools serotonin increases over the night, while among those with fewer days of loose stools it is stable over the night. Anthranilic acid levels are associated with harder stools (Supplementary Table 1).

\section{Exploratory analyses of subjective sleep measures in ir- ritable bowel syndrome group}

The slope of melatonin over the night correlated with several self-reported measures, with better sleep quality associated with a steeper decrease in melatonin (Supplementary Table 2). This is il- 
lustrated in Figure 3C which shows that those who report feeling more rested when they awake in the morning have higher melatonin at Time T1, followed by a steeper decline over the night. Several self-reported measures of sleep quality correlated with the mean and/or the slope of indole-3-acetic acid (Supplementary Table 2). For example, individuals who reported a greater amount of nocturnal waking had significantly higher indole-3-acetic acid throughout the night (Supplementary Fig. 3A).

Usual bed time and awakening time from daily diary are negatively associated with the mean of several metabolites (Supplementary Table 2). Compared to IBS subjects who typically go to bed early and get up early, those who stay up late and sleep later in the morning have lower mean levels of most of the metabolites, with this trend being statistically significant for melatonin, tryptamine, and indole-3-acetic acid. An example is illustrated in Figure 3D for tryptamine. Usual length of sleep was related to a number of metabolites (Supplementary Table 2). As illustrated in Figure 3E, indole-3-lactic acid was notably higher during the second half of the night in participants who typically slept less than 7.5 hours.

\section{Exploratory analyses of polysomnographic data in irri- table bowel syndrome group}

There were few significant associations between PSG variables and either metabolite levels or changes over the night (slope) (Supplementary Table 3). The mean melatonin was significantly higher in participants who slept longer than 455 minutes (Supplementary Fig. 3D). The slope of melatonin was positively associated with slow wave sleep $(P<0.01)$. Indole pyruvate levels were higher $(P$ $<0.05)$ in those who experienced more than $15 \%$ of their sleep time in REM sleep (Fig. 3F).

\section{Exploratory analyses of cortisol, adrenocorticotropic hormone, and cortisol/adrenocorticotropic hormone in irritable bowel syndrome group}

Higher levels of cortisol were inversely associated with Trp ( $r$ $=-0.39)$ and nicotinic acid $(r=-0.38)$ (Supplementary Table 4). Higher ACTH levels were positively associated with a number of metabolites including serotonin $(r=0.35)$, kynurenine $(r=$ $0.49)$, kynurenic acid ( $r=0.73)$, nicotinic acid $(r=0.34)$, and nicotinamide $(r=0.44)$. The ratio of cortisol/ACTH was inversely related to $\operatorname{Trp}(r=-0.44)$, kynurenine $(r=-0.50)$, kynurenic acid $(r=-0.58)$, nicotinic acid $(r=-0.42)$, and indole-3-acetic acid $(r$ $=-0.41)$ (Supplementary Table 4).

\section{Cross correlations among Trp metabolites}

Within the kynurenine and serotonin-melatonin pathways there were many significant positive associations among metabolites (Supplementary Table 5). With the exception of mean indole3-lactic acid with kynurenine, there were no significant relationships between mean serum indole metabolites and the kynurenine and serotonin-melatonin pathway metabolites. With the exception of anthranilic acid, nicotinamide, and indole pyruvate, the slope of Trp across the night was positively correlated with the slopes of its metabolites (Supplementary Table 5).

\section{Discussion}

Self-report of poor sleep is common in persons with $\mathrm{IBS}^{23} \mathrm{We}$ applied targeted, pathway specific MS-based metabolic profiling to describe and analyze Trp metabolite patterns across the night in women with IBS and HCs subjected to a stressor, anticipation of public speaking. We were able to measure 13 of the 20 targeted Trp metabolites. Ten of the thirteen metabolites showed highly significant decreases over the night, and one, nicotinamide, showed a highly significant increase over the night. Omnibus testing of the Trp metabolite profiles found statistically significant differences between IBS and HC groups. Two of the 13 metabolites, nicotinamide and indole-3-lactic acid, demonstrated differences between IBS and HCs (Table 2). Exploration of relationships among metabolite levels and their changes over the night and GI and sleep variables yielded significant associations of melatonin with sleep timing, serotonin and indole with stool form, kynurenine/kynurenic acid with hypothalamic pituitary adrenal (HPA) hormones, and indole-3-acetic acid with sleep variables.

Nicotinamide is an important end metabolite of the Trpkynurenine pathway. It is a substrate for nicotinamide phosphoribosyltransferase (NAMPT) leading to production of nicotinamide adenosine dinucleotide (NAD). Time of day and diurnal changes in NAMPT activity influence nicotinamide levels in urine and blood. ${ }^{24-26}$ Our finding is consistent with observations of Shibata et al, where NAMPT activity was lower during sleep. ${ }^{26}$ In addition, sleep deprivation can disrupt or phase shift the diurnal rhythm of NAMPT activity. ${ }^{27}$ It may be that the higher nicotinamide levels noted in mid-late sleep in women with IBS represents an inherent phase or behavioral difference between groups. The IBS group reported earlier bedtimes and wake times suggesting that lifestyle contributes to the earlier rise in nicotinamide levels. Whether sleep timing behaviors have relevance to IBS is unknown. Our findings suggest that information about sleep behaviors/patterns should be considered when doing metabolite studies. 
Indole-3-lactic acid levels tended to be lower in IBS as compared to HCs (Table 2). Our results are similar to Nagy-Szakal et $\mathrm{al}^{28}$ who compared persons with myalgic encephalomyelitis/chronic fatigue syndrome (ME/CFS) phenotype to HCs. Approximately half of the ME/CFS group self-reported IBS. Examination of women-only revealed that lower indole-3-lactic acid levels distinguished the ME/CFS from HCs. Blood levels of indole-3-lactic acid reflect bacterial as well as diet and liver metabolism. The study of bacterial Trp-indole metabolism and their contribution to serum levels is in its nascent stage. Many bacteria contain enzymes necessary for Trp metabolism. Lower indole-3-lactic acid and higher (albeit not statistically significant) indole-3-acetic-acid levels in the IBS group may reflect differences in gut taxa and/or liver metabolism. There are plausible mechanisms whereby indole metabolites may contribute to IBS pathophysiology, such as activation of argyl hydrocarbon receptor and subsequent inflammation and afferent pain fiber stimulation. ${ }^{29,30}$ However, validation of these mechanisms was beyond the scope of the present study.

Because of its importance in sleep we conjectured that melatonin would either be lower and/or its pattern across the night would be different in the IBS as compared to HCs. While no group differences in melatonin levels were found, the slope of melatonin change across the night was associated with a higher PSQI score and daily poor sleep reports. Those whose melatonin levels decreased more rapidly also reported more abdominal pain and pain after eating. In animal and human studies, pharmacologic melatonin reduces visceral sensitivity. ${ }^{12,31}$ Whether and how melatonin degradation contributes to the link between poor sleep and pain symptoms requires further rigorous study. To clarify these possibilities causal experiments are needed. A study protocol such as that used by Labus and colleagues $^{32}$ in which participants were exposed to acute tryptophan depletion and brain connectivity patterns examined in response to visceral stimuli may reveal insights regarding the role of peripheral Trp on central networks including those involved in sleep.

Based on the literature, serum melatonin levels were expected to peak at Time T3, 2 to 4 hours post sleep onset. ${ }^{33-35}$ However, in both groups melatonin levels were higher at T1 and T2. Because the public speaking stressor produced elevations in cortisol early in the night, ${ }^{5}$ it may be that HPA axis activation and arousal prior to sleep blunted the expected rise in melatonin at Time T3. Although we did find a negative association between cortisol and the ratio of cortisol/ACTH with Trp, there was no association with melatonin.

The best known of the Trp metabolites for its role in IBS is serotonin. Serotonin is involved in neurotransmission and influences visceral pain sensitivity, motility and secretion. ${ }^{36-38}$ We found no IBS versus HC difference in serotonin levels during the night or differences across the night in either group. Serotonin levels did not change markedly across the night. This may be because the enterochromaffin cells synthesize and release serotonin. Also, certain colonic bacteria likely contribute to serotonin levels either through de novo synthesis or the decarboxylation of Trp. Gut bacteria can interact with the enterochromaffin cells to stimulate synthesis of serotonin. ${ }^{39}$ Within the IBS group the slope of peripheral serotonin decrease across the night was positively associated with daily diarrhea, looser stools, more stools, and abdominal pain after eating (Supplementary Table 1). Studies that examine gut taxa may elucidate the contribution of bacteria-host interaction to circulating serotonin levels.

Serum kynurenine and kynurenic acid levels did not differ between IBS and HCs. Although kynurenic acid has been shown to decrease motility index in models of intestinal inflammation, we found no significant relationships between these metabolites and bowel symptoms in the IBS group. These results differ from those of Fitzgerald who reported that IBS participants who rated their symptoms as severe as compared to $\mathrm{HC}$ s and IBS with less severe symptoms had higher morning levels of plasma kynurenine to Trp ratio. ${ }^{40}$ Time of sample collection and the presence of a stressor in our study may explain these differences.

Stress and activation of the HPA axis result in greater activation of the Trp-kynurenine pathway. ${ }^{41,42}$ While we found strong relationships between the ACTH and ratio of cortisol/ACTH and kynurenine and kynurenic acid in the IBS group, similar relationships of these HPA hormones were present with serotonin and melatonin. Although stress is also linked with poor sleep, ${ }^{43}$ we failed to find marked relationships among sleep variables and kynurenine levels. These results are consistent with Baratta et $\mathrm{al}^{44}$ who found no differences in plasma kynurenine and kynurenic acid in female rats exposed to sleep deprivation and stress. However, we did find a significant inverse relationship between kynurenic acid and sleep efficiency. The significance of this finding remains to be determined.

This study has several important limitations. Beyond restricting caffeine and alcohol consumption, no effort was made to control for dietary intake prior to sleep study. All subjects refrained from eating beginning at 20:00 hours. Only women between ages of 20-47 were enrolled. Gender differences in serum Trp have been reported. ${ }^{45}$ Third, we did not assess chronotype characteristics or time the laboratory assessment according to the participants' sleep preferences. Fourth, there might be other factors such as gut taxa, which may have affected sleep measures. ${ }^{46}$ Further, we also did not assess and control for the possible impact of changing participants' normal 
sleep environments on our study outcomes. Finally, the sample size of this project, although typical of multi-night laboratory-based sleep research, was small relative to the number and complexity of the study variables, limiting the statistical power and confidence in the broader generalizability of the reported results. The observational study design allowed us to see intriguing patterns, but did not permit us to address fundamental questions about the causal mechanisms of metabolite action, an important topic for future research.

In summary, this exploratory metabolomic study reveals overall IBS versus HC differences in nocturnal serum Trp metabolites with anticipation of public speaking. Ten Trp metabolites show a strong decrease over the night and 2 metabolites demonstrate IBS versus HC differences. Additional studies including gut bacteria taxa in concert with symptoms and sleeping behaviors are needed to unravel the link between sleep and IBS symptoms.

\section{Supplementary Materials}

Note: To access the supplementary tables and figures mentioned in this article, visit the online version of Journal of Neurogastroenterology and Motility at http://www.jnmjournal.org/, and at https://doi.org/10.5056/jnm19042.

Financial support: This work was supported by grants from NINR NIH (RO1 NR15117, RO1 NR01094, and P30 NR016585).

\section{Conflicts of interest: None.}

Author contributions: Margaret Heitkemper: blood sample collection study and the nocturnal tryptophan metabolites analysis study, and supervised and participated in all aspects of project, from conception to results interpretation and manuscript generation; Kevin Cain (biostatistician) and Robert L Burr (bioengineer): participated in the project teams for both the blood sample collection study and the metabolomics analysis study, participated centrally in study design data analysis, and manuscript development, with special emphasis on the technical aspects of the analysis; Daniel Raftery (Director of the Northwest Metabolomics Research Center) guided the Center team designing and executing the LC-MS experiments targeting the tryptophan metabolites, and worked closely with the IBS researchers to interpret the LC-MS findings; Haiwei Gu, Danijel Djukovic, and Xinyu Zhang were charged with developing and executing the mass spectrometry experiments used in the project; Haiwei $\mathrm{Gu}$ also produced the initial technical draft of the results section; Claire Han participated in discussions about the data analyses and results interpretation and prepared tables and graphs, and edited the manuscript; and Nini Callan participated in discussions about the findings, notably the interpretation of the metabolite profiles and clinical implications, and commented on the manuscript at various stages of its development.

\section{References}

1. Chang L, Di Lorenzo C, Farrugia G, et al. Functional bowel disorders: a roadmap to guide the next generation of research. Gastroenterology 2018;154:723-735.

2. Fass R, Fullerton S, Tung S, Mayer EA. Sleep disturbances in clinic patients with functional bowel disorders. Am J Gastroenterol 2000;95:11952000.

3. Jarrett M, Heitkemper M, Cain KC, Burr RL, Hertig V. Sleep disturbance influences gastrointestinal symptoms in women with irritable bowel syndrome. Dig Dis Sci 2000;45:952-959.

4. Burr RL, Jarrett ME, Cain KC, Jun SE, Heitkemper MM. Catecholamine and cortisol levels during sleep in women with irritable bowel syndrome. Neurogastroenterol Motil 2009;21:1148-e97.

5. Heitkemper MM, Cain KC, Deechakawan W, et al. Anticipation of public speaking and sleep and the hypothalamic-pituitary-adrenal axis in women with irritable bowel syndrome. Neurogastroenterol Motil 2012;24:626-631, e270-e271.

6. Badawy AA, Namboodiri AM, Moffett JR. The end of the road for the tryptophan depletion concept in pregnancy and infection. Clin Sci (Lond) 2016;130:1327-1333.

7. Palego L, Betti L, Rossi A, Giannaccini G. Tryptophan biochemistry: structural, nutritional, metabolic, and medical aspects in humans. J Amino Acids 2016;2016:8952520.

8. Baratta AM, Viechweg SS, Mong JA, Pocivavsek A. A high-performance liquid chromatography measurement of kynurenine and kynurenic acid: relating biochemistry to cognition and sleep in rats. J Vis Exp Publiched Online: 19 Aug 2018. Doi: 10.3791/58129.

9. Keszthelyi D, Troost FJ, Jonkers DM, et al. Does acute tryptophan depletion affect peripheral serotonin metabolism in the intestine? Am J Clin Nutr 2012;95:603-608.

10. Heitkemper MM, Han CJ, Jarrett ME, et al. Serum tryptophan metabolite levels during sleep in patients with and without irritable bowel syndrome (IBS). Biol Res Nurs 2016;18:193-198.

11. Esteban-Zubero E, López-Pingarrón L, Alatorre-Jiménez MA, et al. Melatonin's role as a co-adjuvant treatment in colonic diseases: a review. Life Sci 2017;170:72-81.

12. Song GH, Leng PH, Gwee KA, Moochhala SM, Ho KY. Melatonin improves abdominal pain in irritable bowel syndrome patients who have sleep disturbances: a randomised, double blind, placebo controlled study. Gut 2005;54:1402-1407.

13. Gevi F, Zolla L, Gabriele S, Persico AM. Urinary metabolomics of young Italian autistic children supports abnormal tryptophan and purine metabolism. Mol Autism 2016;7:47.

14. Bryn V, Verkerk R, Skjeldal OH, Saugstad OD, Ormstad H. Kynuren- 
ine pathway in autism spectrum disorders in children. Neuropsychobiology 2017;76:82-88

15. Brooks AK, Lawson MA, Smith RA, Janda TM, Kelley KW, McCusker RH. Interactions between inflammatory mediators and corticosteroids regulate transcription of genes within the kynurenine pathway in the mouse hippocampus. J Neuroinflammation 2016;13:98.

16. Yamashita M, Yamamoto T. Tryptophan and kynurenic acid may produce an amplified effect in central fatigue induced by chronic sleep disorder. IntJ Tryptophan Res 2014;7:9-14.

17. Wu Y, Mai N, Zhong X, et al. Kynurenine pathway changes in late-life depression with memory deficit. Psychiatry Res 2018;269:45-49.

18. Whitehead WE, Drossman DA. Validation of symptom-based diagnostic criteria for irritable bowel syndrome: a critical review. Am J Gastroenterol 2010;105:814-820.

19. Buysse DJ, Reynolds CF 3rd, Monk TH, Berman SR, Kupfer DJ. The pittsburgh sleep quality index: a new instrument for psychiatric practice and research. Psychiatry Res 1989;28:193-213.

20. Backhaus J, Junghanns K, Broocks A, Riemann D, Hohagen F. Test-retest reliability and validity of the Pittsburgh sleep quality index in primary insomnia. J Psychosom Res 2002;53:737-740.

21. Armitage R, Hoffmann R, Fitch T, Morel C, Bonato R. A comparison of period amplitude and power spectral analysis of sleep EEG in normal adults and depressed outpatients. Psychiatry Res 1995;56:245-256.

22. Drewes AM, Nielsen KD, Taagholt SJ, Bjerregård K, Svendsen L, Gade J. Sleep intensity in fibromyalgia: focus on the microstructure of the sleep process. Br J Rheumatol 1995;34:629-635.

23. Tu Q, Heitkemper MM, Jarrett ME, Buchanan DT. Sleep disturbances in irritable bowel syndrome: a systematic review. Neurogastroenterol Motil Published Online First: 28 Sep 2016. Doi: 10.1111/nmo. 12946.

24. Maiese K, Chong ZZ, Hou J, Shang YC. The vitamin nicotinamide: translating nutrition into clinical care. Molecules 2009;14:3446-3485.

25. Bae SA, Androulakis IP. The synergistic role of light-feeding phase relations on entraining robust circadian rhythms in the periphery. Gene Regul Syst Bio 2017;11:1177625017702393.

26. Shibata K. Organ co-relationship in tryptophan metabolism and factors that govern the biosynthesis of nicotinamide from tryptophan. J Nutr Sci Vitaminol 2018;64:90-98.

27. Benedict C, Shostak A, Lange T, et al. Diurnal rhythm of circulating nicotinamide phosphoribosyltransferase (Nampt/visfatin/PBEF): impact of sleep loss and relation to glucose metabolism. J Clin Endocrinol Metab 2012;97:E218-E222.

28. Nagy-Szakal D, Barupal DK, Lee B, et al. Insights into myalgic encephalomyelitis/chronic fatigue syndrome phenotypes through comprehensive metabolomics. Sci Rep 2018;8:10056.

29. Alexeev EE, Lanis JM, Kao DJ, et al. Microbiota-derived indole etabolites promote human and murine intestinal homeostasis through regulation of interleukin-10 receptor. Am J Pathol 2018;188:1183-1194.

30. Gao J, Xu K, Liu H, et al. Impact of the gut microbiota on intestinal immunity mediated by tryptophan metabolism. Front Cell Infect Microbiol 2018;8:13.
31. Borsani E, Buffoli B, Bonazza V, Reiter RJ, Rezzani R, Rodella LF Single administration of melatonin modulates the nitroxidergic system at the peripheral level and reduces thermal nociceptive hypersensitivity in neuropathic rats. Int J Mol Sci 2017;18:2143.

32. Labus JS, Mayer EA, Jarcho J, et al. Acute tryptophan depletion alters the effective connectivity of emotional arousal circuitry during visceral stimuli in healthy women. Gut 2011;60:1196-1203.

33. Daugaard S, Garde AH, Bonde JPE, et al. Night work, light exposure and melatonin on work days and days off. Chronobiol Int 2017;34:942955.

34. Claustrat B, Leston J. Melatonin: physiological effects in humans. Neurochirurgie 2015;61:77-84.

35. Almeneessier AS, Bahammam AS, Sharif MM, et al. The influence of intermittent fasting on the circadian pattern of melatonin while controlling for caloric intake, energy expenditure, light exposure, and sleep schedules: a preliminary report. Ann Thorac Med 2017;12:183-190.

36. Costedio MM, Hyman N, Mawe GM. Serotonin and its role in colonic function and in gastrointestinal disorders. Dis Colon Rectum 2007;50:376-388.

37. Hasler WL. Serotonin and the GI tract. Curr Gastroenterol Rep 2009;11:383-391.

38. Stasi C, Bellini M, Gambaccini D, et al. Neuroendocrine dysregulation in irritable bowel syndrome patients: a pilot study. J Neurogastroenterol Motil 2017;23:428-434.

39. Yano JM, Yu K, Donaldson GP, et al. Indigenous bacteria from the gut microbiota regulate host serotonin biosynthesis. Cell 2015;161:264-276.

40. Fitzgerald P, Cassidy Eugene M, Clarke G, et al. Tryptophan catabolism in females with irritable bowel syndrome: relationship to interferongamma, severity of symptoms and psychiatric co-morbidity. Neurogastroenterol Motil 2008;20:1291-1297.

41. Michels N, Clarke G, Olavarria-Ramirez L, et al. Psychosocial stress and inflammation driving tryptophan breakdown in children and adolescents: a cross-sectional analysis of two cohorts. Psychoneuroendocrinology 2018;94:104-111.

42. Fuertig R, Azzinnari D, Bergamini G, et al. Mouse chronic social stress increases blood and brain kynurenine pathway activity and fear behaviour: both effects are reversed by inhibition of indoleamine 2,3-dioxygenase. Brain Behavior Immu 2016;54:59-72.

43. Lei WY, Chang WC, Wong MW, et al. Sleep disturbance and its association with gastrointestinal symptoms/diseases and psychological comorbidity. Digestion 2019;99:205-212.

44. Baratta AM, Buck SA, Buchla AD, et al. Sex differences in hippocampal memory and kynurenic acid formation following acute sleep deprivation in rats. 2018;8:6963.

45. Hestad KA, Engedal K, Whist JE, Farup PG. The relationships among tryptophan, kynurenine, indoleamine 2,3-dioxygenase, depression, and neuropsychological performance. Front Psychol 2017;8:1561.

46. Li Y, Hao Y, Fan F, Zhang B. The role of microbiome in insomnia, circadian disturbance and depression. Front Psychiatry 2018;9:669. 